\title{
Exercise-Induced Pulmonary Hypertension: How to Define, Diagnose, and Treat At-Risk Patients
}

\author{
Brandon Shokoples $^{1}$ | Kevin Comeau ${ }^{1}$
}

\author{
${ }^{1}$ McGill University

\section{Correspondence} \\ Brandon Shokoples \\ Email: brandon.shokoples@mail.mcgill.ca \\ Fonds de recherche Santé Quebec (FRQS) \\ bourse 289184; Lady Davis Institute/TD \\ Bank Studentship award; Canadian \\ Institutes of Health Research (ClHR) \\ Canada Graduate Scholarship; McGill \\ Department of Medicine Gordon Phillips \\ Fellowship
}

\section{Publication Date}

December 3, 2021

MJM 2022 (20) 14

https://doi.org/10.26443/mjm.v20i1.900

\section{के McGill \\ Journal of Medicine}

www.mjmmed.com

\section{(c) (i) (2)(2)}

This work is licensed under a Creative Commons BY-NC-SA 4.0 International License.

\section{ABSTRACT}

Introduction: Pulmonary hypertension $(\mathrm{PH})$ is a devastating disease that progresses rapidly, leading to high patient mortality. This condition is characterized by high blood pressure in the pulmonary vasculature and poor pulmonary perfusion, resulting in patient fatigue, dyspnea, and syncope-especially upon physical exertion. A sub-clinical form of $\mathrm{PH}$ is referred to as exercise induced pulmonary hypertension (EIPH), where patients display normal resting hemodynamic properties but abnormal pulmonary responses to exercise.

Discussion: Recent evidence suggests that early intervention and treatment of $\mathrm{PH}$ can improve patient outcomes. However, little clinical evidence exists to support effective treatments of EIPH. This lack is due in part to the removal of EIPH from official guidelines, such as the European Respiratory Society in 2008. EIPH was removed from clinical guidelines because of a lack of consensus on what constitutes EIPH and a lack of agreement on standardized testing procedures for diagnosing EIPH. Emerging evidence suggests that exercise testing following a standardized protocol of stress echocardiography or right heart catheterization may allow for better classification of EIPH. This review proposes that a mean pulmonary artery pressure/cardiac output slope $>3 \mathrm{~mm} \mathrm{Hg} / \mathrm{L} / \mathrm{min}$ or mean pulmonary artery pressure $>30 \mathrm{~mm} \mathrm{Hg}$ with a pulmonary vascular resistance $>3$ Wood units is both a sensitive and specific enough threshold to diagnose EIPH and is sufficient to consider specific treatment options.

Conclusion: Providing evidence for a consensus on the definition of EIPH, along with a validated and standardized testing procedure, will hopefully foster the progression of research on EIPH and further the development of treatments to improve patient outcomes.

\section{KEYWORDS}

Pulmonary hypertension, Exercise-induced pulmonary hypertension, Cardiopulmonary exercise testing 


\section{1 | INTRODUCTION}

Pulmonary hypertension $(\mathrm{PH})$ is a relatively rare yet devastating disease that is estimated to affect $1 \%$ of the global population under 65 and $10 \%$ over the age of 65. (1) PH is a progressive disease that results in decreasing exercise intolerance, dyspnea, fatigue, altered hemodynamic and cardiopulmonary function, and low long-term survival rates. $(2,3)$ If left untreated, the average length of survival for patients is approximately three years from the time of diagnosis. This delay is of concern because the average time from patient-described onset of $\mathrm{PH}$ symptoms to diagnosis of the disease is approximately 4 years. (4) In recent years, the prognosis for $\mathrm{PH}$ patients has been improved by the advent of new and improved therapies like pulmonary vasodilators and diuretics; however, a diagnosis of $\mathrm{PH}$ in Canada still increases the 1-year standardized mortality ratio by 7.2fold. (5) Recent studies suggest that interventions conducted at earlier stages, or even pre-clinical stages of $\mathrm{PH}$, may result in increased longevity and quality of life for patients. $(6,7)$ Therefore, a need exists for improved diagnostic methods to identify and treat patients with $\mathrm{PH}$ early in the development of the disease.

$\mathrm{PH}$ is characterized by abnormal pulmonary hemodynamics and high pulmonary blood pressure. This increased pulmonary blood pressure can be the result of several different underlying pathologies; however, the increase is typically caused by an obstruction to flow downstream of the right ventricle, either in the pulmonary arteries or the aortic valve. (8) Recently, the definition of $\mathrm{PH}$ was updated to be a resting mean pulmonary arterial pressure (mPAP) of $20 \mathrm{mmHg}$ with a pulmonary vascular resistance (PVR) of 3 Wood units. (9) This new definition replaces the previous arbitrary cut-off of a mPAP $25 \mathrm{mmHg}$ to include two standard deviations above the mean mPAP of $14 \mathrm{mmHg}$ in healthy people. However, many people who have otherwise normal resting hemodynamics, display dyspnea and fatigue during moderate to mild exercise. When investigating the source of this exertional intolerance, researchers discovered that many of these people in fact had severely elevated mPAP and/or PVR while exercising. When tracked, a large proportion of these patients were found to develop abnormal resting hemodynamics, especially when other comorbidities like heart failure, systemic sclerosis, or scleroderma are present. (10-13) This observation led many people to believe that an intermediate step exists between normal pulmonary pressure and overt $\mathrm{PH}$, which is referred to as exercise induced pulmonary hypertension (EIPH). (7, 14-18).

Until 2008, EIPH had been included in the official European Society of Cardiology and European Respiratory Society guidelines for PH. EIPH was described as a mPAP exceeding $30 \mathrm{mmHg}$, a pulmonary artery systolic pressure $(\mathrm{PASP})>50 \mathrm{mmHg}$, or a pulmonary capillary wedge pressure (PCWP) $<20 \mathrm{mmHg}$ during exercise. (8) However, in 2008, the official guidelines for pulmonary hypertension diagnosis and treatment removed EIPH as a subset of $\mathrm{PH}$. (8) The old definition of EIPH was abandoned because of its failure to accurately and consistently differentiate healthy patients from those experiencing true EIPH. Failure to differentiate healthy individuals was especially true in patients with increased cardiac outputs (CO), as their mPAP values would often exceed the $30 \mathrm{~mm} \mathrm{Hg}$ threshold established for EIPH. (19, 20 ) In one example, a study found that $26 \%$ of healthy patients displayed a mPAP > $30 \mathrm{~mm} \mathrm{Hg}$ with exercise, especially as COs exceeded $10 \mathrm{~L} / \mathrm{min}$. (21) Another issue that resulted in the old definition being abandoned was disparity in the methodology and exertion metrics used to diagnose EIPH. (22) Some researchers proposed that stress echocardiography (SE) measurements during exercise were sufficient to diagnose EIPH, while others, including the Canadian Thoracic Society maintained that right heart catherization (RHC) was necessary for diagnosis. $(11,23)$ Additionally, the formulas used to attain pulmonary pressure and vascular resistance varied between groups, as well as the actual exercise parameters used (ie, cycle ergometry vs treadmill tests). Therefore, consistency and accuracy in measurements was a major concern that ultimately forced the definition to be abandoned.

More recently, new thresholds for defining EIPH have been put forth; these new thresholds are demonstrated to be more sensitive and specific for defining EIPH. The 
improvement of diagnostic techniques and methodology has led to more consistent measurements of hemodynamic properties. The definitions that were recently suggested for EIPH are a mPAP to CO slope of $>3 \mathrm{~mm}$ $\mathrm{Hg} / \mathrm{L} / \mathrm{min}(24,25)$ or a mPAP $>30 \mathrm{~mm} \mathrm{Hg}$ with total peripheral resistance $>3$ Wood units during exercise. (21) Since EIPH is viewed as a potentially more treatable stage of $\mathrm{PH}$ it is crucial to develop a standard diagnostic approach with reliable threshold parameters to accurately diagnose EIPH. (17) This review will briefly describe the etiology of $\mathrm{PH}$ and focus on creating a definition for EIPH, illustrating the gold standards for diagnosis, and discussing relevant interventions for patients.

\section{2 | ETIOLOGY OF PH}

$\mathrm{PH}$ is a progressive disease of abnormal pulmonary hemodynamics and increased pulmonary circulation pressures. (14) Currently there are 5 different clinical classifications of PH (Table 1) with different subclassifications based upon the mechanisms of onset. (9) The two most common causes of increased pulmonary pressure are increased PVR and increased left atrial pressure (LAP) $(13,26)$ In many cases, the initial onset of PH is idiopathic, however, the progression of EIPH typically stems from changes in the pulmonary vasculature that induce inflammation, vasoconstriction, cell proliferation, and hypertrophy. The result is exacerbated pulmonary damage inducing strain and dysfunction of the right ventricle (RV). (27)

It is important to try to understand the underlying etiology of the condition as it can help guide the treatment approach and the success of the therapy. For conditions with a known etiology, the treatment is often adapted to address the underlying cause of the $\mathrm{PH}$. For example $\mathrm{PH}$ due to left heart disease is often treated with a mechanical intervention such as surgery to repair the defect (i.e. a valve replacement for a valvular disease). (8) For other etiologies where a mechanical intervention is not obvious or relevant, the endothelin (ET), nitric oxide (NO), and prostacyclin (PC) dependent pathways are currently being utilized as therapeutic targets in Canada. (23) ET activates two different receptor subtypes (ETA and ETB) present on vascular smooth muscle cells (vSMC) and endothelial cells (EC) (ETB only). Activation of ET receptors on vSMCs induces vasoconstriction, cellular proliferation, and hypertrophy, while activation on ECs results in vasodilation. (28) In PH it is believed there is a shift increasing ET expression on vSMCS that results in the deleterious phenotype associated with $\mathrm{PH}$; therefore, ET antagonists such as Ambrisentan have been employed with relative success. $(23,29,30) \mathrm{NO}$ is a potent vasodilator that also inhibits platelet aggregation and thrombosis. Under normal conditions, ECs continuously produce NO using nitric oxide synthase (NOS), causing relaxation of vSMCs and inhibition of cellular proliferation. $(31,32)$ In $\mathrm{PH}$, levels of NOS are greatly reduced resulting in an increased vasoconstriction and cellular hypertrophy. (29) Prostacyclin (PC) is produced in ECs through prostacyclin synthase and acts as a potent vasodilator. (27) PC binds to prostaglandin receptors and induces relaxation of vSMCs. In PH PC levels are diminished, contributing to the phenotype of vasoconstriction and cellular hypertrophy observed in $\mathrm{PH}$. (33) Since all three of these pathways contribute to rising PVR and increased pulmonary pressures, they have been investigated extensively and make up the bulk of therapeutic targets for patients suffering from $\mathrm{PH}$. (8, 23)

\section{MEASURING EIPH AND COM- MON TOOLS USED FOR DIAGNO- SIS}

A common complaint of patients with undiagnosed $\mathrm{EIPH}$ or $\mathrm{PH}$ is exertional fatigue/dyspnea. Therefore, SE is a common starting diagnostic procedure followed by chest $x$-rays, electrocardiograms (EKG), echocardiograms (ECG), chest CT or MRI scans, and blood tests to identify the underlying cause and rule out conditions other than PH. (14) As exertional dyspnea/fatigue is the most frequent presenting symptom, it is often useful to attempt diagnosis of EIPH or $\mathrm{PH}$ using cardiopulmonary exercise testing (CPX). (22) However, some con- 
cerns have been raised about making patients with compromised cardiopulmonary systems complete exercise testing. Despite concerns, a comprehensive study involving over 4000 patients who underwent CPX while presenting with high risk cardiac diseases found an adverse event rate of only $0.16 \%$. (34) Looking at the 194 patients with $\mathrm{PH}$ in the study, there were 0 adverse events after $\mathrm{CPX}$ in $\mathrm{PH}$ patients providing reasonable proof of the safety of $\mathrm{CPX}$ in $\mathrm{PH}$ patients. CPX usually includes monitoring ventilatory-perfusion parameters along with right and left heart functioning either through non-invasive ECG or invasive RHC. $(16,22)$ From these tools, values of CO, PVR, PCWP (which estimates left atrial pressure), right ventricle regurgitation velocity, mPAP, PASP, ventricular elasticity and wall thickness, right atrial pressure, peak VO2, lactic acidosis, CO2 output, oxygen saturation, and minute ventilation (VE/VCO2) can be generated and used to assess the presence and extent of the progression of disease.

\section{CHALLENGES IN DIAGNOSING IN EIPH}

Many of the parameters used to diagnose EIPH are estimated from derivations of the following formula for mPAP, assuming an ohmic linear relationship between the parameters. (22)

$$
m P A P=P V R * C O+L A P
$$

The assumption being that any changes in PVR or $\mathrm{CO}$ will result in linear increases of $\mathrm{MPAP}$, and that increases in LAP through backflow will increase MPAP in a 1:1 ratio. However, this does not consider the dynamic responses of the pulmonary vasculature in responses to changes in pressure. In normal healthy individuals, pressure increases are quickly met with pulmonary dilation to normalize flow. Therefore, past predictions of mPAP from this formula may be inaccurate. Herve and colleagues (21) demonstrated that this formula for PVR only provided a $48 \%$ sensitivity, unless the patient had a purely vascular etiology (implying limited distensibility of the vasculature leading to a more linear relation). A more comprehensive alternative formula has been proposed to take into account the distensibility of the venous system. $(22,35)$

$$
m P A P=\frac{(1+\alpha L A P)^{5}+(5 \alpha R o * Q)^{\frac{1}{5}}-1}{\alpha}
$$

Where Ro is the resting total pulmonary resistance, as defined by the ratio of $\mathrm{mPAP} / \mathrm{CO}, \mathrm{Q}$ is pulmonary blood flow and is the distensibility coefficient of the vasculature. The coefficient can be obtained by measuring the pulse pressure of a large artery, but is often assumed as $2 \%$ in most species. (22) This formula better represents the pressure flow relation shown in a compliant vasculature and could potentially lead to more accurate mPAP approximations.

Another issue that arises from the way values are commonly derived from functional tests is the calculation of $\mathrm{CO}$. It is often cited in literature that $\mathrm{CO}$ can be approximated equivocally from thermodilution or direct Fick measurements. (36) However, previous CO measurements had only been validated in resting patients and Hsu and colleagues found that using thermodilution actually greatly overestimated the prevalence of EIPH in patients, with a $20 \%$ increase in false diagnoses. (37) As CO increased in patients, the separation in predicted Fick and thermodilution COs increased, with thermodilution underpredicting $\mathrm{CO}$ relative to Fick measurements. This is extremely concerning, given the importance of accurate $\mathrm{CO}$ measurements in predicting other important parameters for diagnosing EIPH, such as total pulmonary resistance and PVR. Therefore, it is possible that the prevalence of EIPH has been overreported in the literature where studies used thermodilution.

Currently, there is a consensus on what constitutes normal resting values for many of the parameters described above, (i.e. mPAP $<20 \mathrm{mmHg}$ ). However, the definitions for what constitutes normal hemodynamic parameters during exercise remain elusive. Especially since some of the techniques used to diagnose $\mathrm{PH}$ at rest have not been well validated for measurements of 
the same parameters during exercise.

\section{1 | Difficulty in consistent measurements}

A major difficulty in diagnosing EIPH is making consistent measurements on patients during exercise. Performing invasive RHC or SE on an actively moving patient can be a difficult procedure. In addition, normalizing the workload between patients to establish a normal PAP is challenging. (16) Often a cycle ergometer is used with patients in an upright position, with work values ranging from $10-30 \mathrm{~W}$; however, other methods have been used such as treadmills or supine leg press. (22) This can lead to disparity in results, since patient stance/posture can have substantial effects on intrathoracic pressure and concomitantly on pulmonary pressure. $(17,38)$ SE is often used as a diagnostic tool as it is readily available in most clinics and is a non-invasive measurement, but SE has not been well validated for use during exercise. (18) One issue with using SE during exercise is that predictions of right atrial pressure often rely on measurements of the distension of the inferior vena cava. However, it is known that exercise lowers venous compliance and therefore can reduce the accuracy of observations made by SE. $(18,39)$ However, performing SE after the patient has finished exercise runs the risk of missing important data, as hemodynamic parameters quickly return to resting levels within five minutes post-exercise. (40) Another issue with SE, is that PCWP, which is a standard measurement in the diagnosis of $\mathrm{PH}$, cannot be calculated without direct invasive measurements such as RHC. Yet invasive measurements are not without their own problems, as they can be difficult to obtain during exercise because of large swings in intrathoracic pressure that accompany heavy breathing during exercise. (16)

\section{2 | Variation in measurements of healthy individuals}

One of the main reasons EIPH was removed from the official guidelines in 2008 , and not reinstated in the updated guidelines released in 2019, was the lack of consensus among experts on what constitutes normal pulmonary pressures during exercise. Initially EIPH was defined as a mPAP>30 $\mathrm{mmHg}$, but multiple studies have found that healthy individuals consistently exceed mPAP values of $30 \mathrm{mmHg}$ during strenuous exercise, especially in well-trained athletes. (19-21) The caveat being that the increased mPAP and PASP seen with these individuals were accompanied by an increased $\mathrm{CO}$, which fits in line with the observation that healthy individuals do not exceed a mPAP/CO slope of $3 \mathrm{mmHg} / \mathrm{L} / \mathrm{min}$. $(19,41)$ As well, studies have shown that $6 \%$ of otherwise healthy individuals over 50 have a PASP $>40 \mathrm{mmHg}$ as well as $5 \%$ of people with a $\mathrm{BMI}>30$. (42) More recent studies have shown that healthy (nonathletes) cannot exceed PASP values of $40-45 \mathrm{mmHg}$ at $\mathrm{CO}<20 \mathrm{~L} / \mathrm{min}$ during exercise. $(41,43)$ However, these studies excluded athletes.

PASP is most often estimated from the following formula:

$$
P A S P=4 V^{2}+R A P
$$

Where $\mathrm{V}$ is the maximum velocity of the tricuspid valve regurgitant jet and RAP is the right atrial pressure. Tricuspid valve regurgitation itself has been suggested as a defining criteria for EIPH as a regurgitant jet velocity of $>3.0 \mathrm{~m} / \mathrm{s}$, but in line with the findings that PASP can often exceed EIPH definitions, researchers found that athletes had an average peak tricuspid regurgitation velocity of $3.41 \mathrm{~m} / \mathrm{s}$. (44) Therefore, the large variations of hemodynamic parameters in healthy individuals and trained athletes adds to the challenge of finding a single parameter to define EIPH.

\subsection{Predictive ability of Different Diagnostic Tools}

The multifactorial nature of $\mathrm{PH}$ requires a large repertoire of diagnostic techniques to pinpoint the etiology of each patient's PH. Several methods that are effective in the initial screening of patients have proven to 
be ineffective at diagnosing EIPH when used in isolation. Electrocardiograms, although useful for providing direction for further investigation, were shown to be inadequate at diagnosing $\mathrm{PH}$ with a sensitivity (true positive rate) of only $55 \%$ and a specificity (true negative rate) of 70\%. (45) Minute ventilation (VE/VCO2) at ventilatory threshold and peak alveolar-arterial difference in PO2 were not sensitive enough to distinguish EIPH from normal. (18) The lack of standardized procedures for SE has led to both over and underestimations of PAP. (3) When compared to RHC, SE displays variable correlation values between 0.57-0.93 with PASP measurements (11) A more recent study found that 35 of 100 patients identified to have EIPH according to SE, actually had normal hemodynamics via RHC results. (15) Therefore caution must be used when interpreting SE results.

The current gold standard for diagnosing EIPH or $\mathrm{PH}$ is RHC. The biggest downfall of RHC is lack of availability and the inconvenience for the patient in using an invasive measurement. For this reason and also for simplicity, SE is often the preferred first method for screening patients of suspected EIPH or PH. $(22,23)$ Despite the limitations of SE, it allows for quick, easy and relatively reliable estimates, when conducted properly, of PASP, PVR, and pericardial effusions, as well as overall morphology of the heart and valves allowing for diagnoses of RV size and function, RA size, diastolic dysfunctions, valvular functions, patent foramen ovale, and intrapulmonary shunts. (45) However, the importance of standardized techniques used during SE to ensure more consistent and better interpretation of results cannot be understated.

\section{5 | PROPOSED STANDARD PRO- TOCOL AND DEFINITION OF EIPH}

Creating a definition for EIPH is challenging as it exists in an intermediary state between normal values and overt $\mathrm{PH}$. Normal hemodynamic values used for diagnosis of $\mathrm{PH}$ have been shown to vary substantially even among healthy control patients during exercise, making it diffi- cult to isolate clear standards for the subset of patients displaying EIPH. The traditional definition of EIPH being a $\mathrm{mPAP}>30 \mathrm{mmHg}$, PASP $>50$, or PCWP $<20 \mathrm{mmHg}$ during exercise, proved to be inefficient at adequately separating the distinct patient populations (healthy vs EIPH vs overt PH). Therefore, efforts have been made to revise and improve upon these thresholds to find parameters that are more sensitive and specific. It seems clear that a singular definition to define EIPH is not reasonable. Instead, a consensus between several parameters should be used for diagnosis. The best and most current thresholds for an EIPH diagnosis is: a mPAP $>30 \mathrm{~mm} \mathrm{Hg}$ with $\mathrm{CO}<10 \mathrm{~L} / \mathrm{min}$ or a mPAP/CO slope $>3 \mathrm{mmHg} / \mathrm{L} / \mathrm{min}$; or a $\mathrm{mPAP}>30 \mathrm{mmHg}$ with a resistance greater than 3 Wood units (Table 2). These thresholds are shown to be $93 \%$ sensitive and $100 \%$ specific. $(21,24,25)$ Several other parameters are useful for unlocking the etiology of EIPH, albeit insufficient for diagnosis. VE/VCO2 can be useful for separating stages of $\mathrm{PH}$, as VE/VCO2, increases as $\mathrm{PH}$ worsens, but is unchanged in EIPH patients compared to normal. (46) Whereas PASP and PCWP can be used as indicators for the presence of heart disease (45) Barst and colleagues describe the ideal testing methodology in their review, from which we propose a standardized methodology to test for EIPH (Figure 1). (45)

\section{6 | SHOULD WE TREAT EIPH}

The lack of a current formally acknowledged definiton for EIPH does not mean the disease does not merit treatment. However, at the time of this review, a limited number of studies have been conducted to investigate the efficacy of treating patients with EIPH. This phenomenon is despite the fact that numerous studies have shown that the earlier the intervention, the better the prognosis for patients with $\mathrm{PH}$. (8, 47-49) $\mathrm{PH}$ therapy has been shown to slow and prevent further disease progression as well as improve hemodynamic properties of the patients. (6) Kovacs et al (47) reported greater decrease in PAP and PVR with intervention earlier in the stages of disease progression (lower WHO functional class) . Ear- 
1. Include patients with exercise intolerance whose resting $\mathrm{mPAP}<20 \mathrm{mmHg}$.

2. Dynamic exercise in supine position on a stress echocardiography bed, if possible, to allow for constant measurement with SE while patient pedals on a cycle ergometer.

3. Use estimated exercise capacity to design number of work steps and work increments to reach maximum tolerable workload within 10-15 minutes.

4. Take baseline measurements while patient is pedaling at a workload of 0 watts.

5. Increase workload increments at constant rate, between 10$30 \mathrm{~W}$.

6. Constantly measure mPAP using peak pulmonary regurgitation Doppler signal.*

7. Take measurements of PCWP, PASP, and CO (through direct FICK measurement).

8. Stop procedure once the thresholds for EIPH have been met ( $\mathrm{mPAP} / \mathrm{CO}$ slope $>3 \mathrm{mmHg} / \mathrm{L} / \mathrm{min}$ or $\mathrm{mPAP}>30 \mathrm{mmHg}+$ TPR $>3 \mathrm{WU}$, or patient reaches maximal tolerable workload.

9. If uncertainty in the results of SE proceed to RHC and repeat steps 2-8.

FIGURE 1 Proposed standardized protocol for exercise hemodynamic testing.

*For a comprehensive review on SE techniques, see reference (51).

lier treatment has also been suggested to lead to less disease morbidity. $(48,49)$ A recent study demonstrated that patients with EIPH (defined as a mPAP/CO slope > $3 \mathrm{mmHg} / \mathrm{L} / \mathrm{min}$ during exercise) had a 2-fold increase in the hazard ratio of a future cardiovascular event or death comapred to patients with a normal mPAP/CO slope during exercise. (50) As EIPH is considered by many to be a distinct and early stage in the progression of $\mathrm{PH}$, it stands to reason that early intervention will delay the progression into classically defined $\mathrm{PH}$, extend the expected lifespan of patients, and greatly improve patients' future quality of life. Not only does early diagnosis lead to a better prognosis but also to more robust treatment options for the patients. Late stages of the disease often require continuous IV administration of drugs, which greatly diminishes patient quality of life. Whereas for earlier stages, patients often receive oral therapies which are less intrusive and more convenient.

Currently, no drugs are specifically tailored for treatment of EIPH; however, some clues can be taken from a study that looked at scleroderma patients displaying normal pulmonary hemodynamics but abnormal exercise hemodynamics at rest. Saggar et al (48) showed that invasive measurements were easily able to distinguish different stages of abnormal hemodynamic progression, and the authors suggested that early treatment of patients with EIPH-like symptoms using a pulmonary vasodilator (ambrisentan) may yield promising results. Many patients whose $\mathrm{PH}$ is derived from pulmonary vascular disorders are prescribed pulmonary vasodilators, and the results from these studies may yield some additional insight into EIPH treatment. The more recent therapy of choice for treatment of early stages of PH are endothelin blockers (ETA and ETB). One such drug currently on the market is bosentan, which has been shown to improve exercise capacity (increased SixMinute Walk Test levels), vascular resistance, and cardiac index. (6) Bosentan was also shown to decrease brain natriuretic peptide levels which along with improved vascular resistance are predictive factors for survival. The most promising characteristic of endothelin blockers so far is the lack of reported adverse sideeffects. The most common reported side-effect has been increased liver amminotransferase levels and has yet to be correlated with adverse events. (6) Other drug targets include prostanoids and phosphodiesterase- 5 inhibitors. However, these therapies are less preferred for treatment of earlier stages of EIPH because they either have more adverse side-effects or call for lessconvenient administration methods, like IV injections. (27)

In Canada, there are currently $10 \mathrm{PH}$-specific treatments that are approved and have signficiantly improved patient quality of life and long-term survival. (23) Despite the promising results of these therapies in treating many conditions, they have proved less effective in treating $\mathrm{PH}$ caused by systolic and/or diastolic dysfunctions. (49) Typically, anti-clotting agents, such as warfarin, and diuretic agents are prescribed to patients with resting $\mathrm{PH}$; however, whether these treatments would be beneficial for patients suffering from EIPH is uncertain. (27) Calcium channel blockers may also be prescribed to $\mathrm{PH}$ patients, but these drugs' safety and effi- 
cacy profiles may not warrant use in patients with EIPH. Finally, dual therapy has been shown to provide an additional advantage when compared to monotherapy, yet again, no evidence exists for efficacy of dual therapy in treating EIPH patients.

In general, very little data supports the efficacy of treating EIPH. Nevertheless, studies evaluating the effect of treating early stages of $\mathrm{PH}$ appear to be advantageous. With the fast progression of $\mathrm{PH}$ and high mortality rate associated with the disease, more studies evaluating the efficacy of EIPH treatment are necessary. As most cases of EIPH are believed to be the result of pulmonary vascular diseases or left heart disease (both of which increase PVR and pulmonary pressure), pulmonary vasodilators and ET antagonists would appear to be the most promising therapy for treating patients with EIPH. $(22,27)$ Consequently, further studies are needed to provide a conclusive argument for the efficacy of treating EIPH.

\section{7 | CONCLUSION}

EIPH has consistently been shown as a distinct entity that is intermediate between normal hemodynamics and overt pulmonary hypertension. However, the lack of consensus on defining criteria for EIPH and an absence of standardized treatment methodologies has led to the poor state of knowledge on treatment efficacy for this subset of patients. With evidence showing that treatment of the early stages of $\mathrm{PH}$ leads to increased longevity and better quality of life for patients, it is crucial to evaluate if these results are transferable to patients suffering from EIPH. This review proposes that a $\mathrm{mPAP} / \mathrm{CO}$ slope $>3 \mathrm{mmHg} / \mathrm{L} / \mathrm{min}$ or $\mathrm{mPAP}>30 \mathrm{mmHg}$ + TPR > 3 Wood units is both a sensitive and specific enough threshold to diagnose EIPH and is sufficient to consider specific treatment options. Having a set EIPH definition for clinicians along with the standardized protocol provided here will allow research to move forward on treatments for EIPH, and hopefully lead to a better future prognosis for patients who develop pulmonary hypertension.

\section{REFERENCES}

1. Hoeper MM, Humbert M, Souza R, Idrees M, Kawut SM, Sliwa-Hahnle K, et al. A global view of pulmonary hypertension. Lancet Respir Med. 2016;4(4):306-22. DOI:10.1016/S22132600(15)00543-3

2. Fowler RM, Maiorana AJ, Jenkins SC, Gain KR, O'Driscoll

G, Gabbay E. Implications of exercise-induced pulmonary arterial hypertension. Medicine and Science in Sports and Exercise. 2011;43(6):983-9. DOI:10.1249/MSS.0b013e318204cdac

3. Steen V, Chou M, Shanmugam V, Mathias M, Kuru T, Morrissey R. Exercise-induced pulmonary arterial hypertension in patients with systemic sclerosis. Chest. 2008;134(1):146-51. DOI:10.1378/chest.07-2324

4. Yamada H, Saijo Y, Hotchi J, Hayashi S, Bando M, Nishio S, et al. Right Ventricular Dysfunction in Patients With ExerciseInduced Pulmonary Hypertension Associated With Connective Tissue Disease. Journal of the American College of Cardiology. 2014;63(12):A1499-A. DOI:10.1016/S0735-1097(14)61500-X

5. Wijeratne DT, Lajkosz K, Brogly SB, Lougheed MD, Jiang $L$, Housin $A$, et al. Increasing Incidence and Prevalence of World Health Organization Groups 1 to 4 Pulmonary Hypertension. Circulation: Cardiovascular Quality and Outcomes. 2018;11(2):e003973. DOI:10.1161/circoutcomes.117.003973

6. Galiè N, Rubin L, Hoeper M, Jansa P, Al-Hiti H, Meyer G, et al. Treatment of patients with mildly symptomatic pulmonary arterial hypertension with bosentan (EARLY study): a double-blind, randomised controlled trial. The Lancet. 2008;371(9630):2093-100. DOI:10.1016/S0140-6736(08)60919-8

7. Proudman SM, Stevens WM, Sahhar J, Celermajer D. Pulmonary arterial hypertension in systemic sclerosis: The need for early detection and treatment. Internal Medicine Journal. 2007;37(7):485-94. DOI:10.1111/j.1445-5994.2007.01370.x

8. Galiè N, Hoeper MM, Humbert M, Torbicki A, Vachiery JL, Barbera JA, et al. Guidelines for the diagnosis and treatment of pulmonary hypertension. European Respiratory Journal. 2009;34(6):1219-63. DOI:10.1183/09031936.00139009

9. Simonneau G, Montani D, Celermajer DS, Denton CP, Gatzoulis MA, Krowka M, et al. Haemodynamic definitions and updated clinical classification of pulmonary hypertension. European Respiratory Journal. 2019;53(1):1801913. DOI:10.1183/13993003.019132018

10. Condliffe R, Kiely DG, Peacock AJ, Corris PA, Gibbs JSR, Vrapi F, et al. Connective tissue disease-associated pulmonary arterial hypertension in the modern treatment era. American Journal of Respiratory and Critical Care Medicine. 2009;179(2):151-7. DOI:10.1164/rccm.200806-9530C

11. Denton CP, Cailes JB, Phillips GD, Wells AU, Black CM, Du Bois RM. Comparison of Doppler Echocardiography and Right Heart Catheterization To Assess Pulmonary Hypertension in Systemic Sclerosis. British Journal of Rheumatology. 1997;36:239-43. DOI:10.1093/rheumatology/36.2.239 
12. Obokata M, Nagata $\mathrm{Y}$, Kado $\mathrm{Y}$, Kurabayashi M, Otsuji Y, Takeuchi M. Ventricular-Arterial Coupling and ExerciseInduced Pulmonary Hypertension During Low-Level Exercise in Heart Failure With Preserved or Reduced Ejection Fraction. Journal of Cardiac Failure. 2017;23(3):216-20. DOI:10.1016/j.cardfail.2016.10.001

13. Voilliot D, Magne J, Dulgheru R, Kou S, Henri C, Laaraibi S, et al. Determinants of exercise-induced pulmonary arterial hypertension in systemic sclerosis. International Journal of Cardiology. 2014;173(3):373-9. DOI:10.1016/j.ijcard.2014.02.042

14. McGoon M, Gutterman D, Steen V, Barst RJ, McCorry D, Fortin T, et al. Screening, Early Detection, and Diagnosis of Pulmonary Arterial Hypertension. Chest. 2004;126(1 Suppl):78S-92S. DOI:10.1378/chest.126.1

15. Misra D, Kendes A, Sulica R, Carabello B. Exercise-induced pulmonary hypertension by stress echocardiography: Prevalence and correlation with right heart hemodynamics. International Journal of Cardiology. 2017;228:518-22. DOI:10.1016/j.ijcard.2016.11.191

16. Oudiz RJ, Rubin LJ. Exercise-induced pulmonary arterial hypertension: A new addition to the spectrum of pulmonary vascular diseases. Circulation. 2008;118(21):2120-1. DOI:10.1161/CIRCULATIONAHA.108.819573

17. Raeside Da, Chalmers G, Clelland J, Madhok R, Peacock aJ. Pulmonary artery pressure variation in patients with connective tissue disease: 24 hour ambulatory pulmonary artery pressure monitoring. Thorax. 1998;53(10):857-62. DOI:10.1136/thx.53.10.857

18. Tolle JJ, Waxman AB, Van Horn TL, Pappagianopoulos PP, Systrom DM. Exercise-induced pulmonary arterial hypertension. Circulation. 2008;118(21):2183-9. DOI:10.1161/CIRCULATIONAHA.108.787101

19. Kovacs G, Berghold A, Scheidl S, Olschewski H. Pulmonary arterial pressure during rest and exercise in healthy subjects: A systematic review. European Respiratory Journal. 2009;34(4):888-94. DOI:10.1183/09031936.00145608

20. Naeije R, Mélot C, Niset G, Delcroix M, Wagner PD. Mechanisms of improved arterial oxygenation after peripheral chemoreceptor stimulation during hypoxic exercise. Journal of Applied Physiology. 1993;74(4):1666-71.

21. Herve P, Lau EM, Sitbon O, Savale L, Montani D, Godinas L, et al. Criteria for diagnosis of exercise pulmonary hypertension. European Respiratory Journal. 2015;46(3):728-37. DOI:10.1183/09031936.00021915

22. Guseh JS. The Evolving Landscape of Exercise-Induced Pulmonary Hypertension. Current Treatment Options in Cardiovascular Medicine. 2016;18(6). DOI:10.1007/s11936-016-0459-5

23. Hirani N, Brunner NW, Kapasi A, Chandy G, Rudski L, Paterson I, et al. Canadian Cardiovascular Society/Canadian Thoracic Society Position Statement on Pulmonary Hypertension. Canadian Journal of Cardiology. 2020;36(7):977-92. DOI:10.1016/j.cjca.2019.11.041

24. Lewis GD, Bossone E, Naeije R, Grünig E, Saggar R, Lancellotti
P, et al. Pulmonary vascular hemodynamic response to exercise in cardiopulmonary diseases. Circulation. 2013;128(13):1470-9. DOI:10.1161/CIRCULATIONAHA.112.000667

25. Naeije R, Vanderpool R, Dhakal BP, Saggar R, Saggar R, Vachiery JL, et al. Exercise-induced pulmonary hypertension: Physiological basis and methodological concerns. American Journal of Respiratory and Critical Care Medicine. 2013;187(6):576-83. DOI:10.1164/rccm.201211-2090CI

26. Maclver DH, Adeniran I, Maclver IR, Revell A, Zhang H. Physiological mechanisms of pulmonary hypertension. American Heart Journal. 2016;180:1-11. DOI:10.1016/j.ahj.2016.07.003

27. McLaughlin VV, Archer SL, Badesch DB, Barst RJ, Farber HW, Lindner JR, et al. ACCF/AHA 2009 Expert Consensus Document on Pulmonary Hypertension. A Report of the American College of Cardiology Foundation Task Force on Expert Consensus Documents and the American Heart Association Developed in Collaboration With the American College o. Journal of the American College of Cardiology. 2009;53(17):1573-619. DOI:10.1016/j.jacc.2009.01.004

28. Sumner MJ, Cannon TR, Mundin JW, White DG, Watts IS. Endothelin ETA and ETB receptors mediate vascular smooth muscle contraction. British Journal of Pharmacology. 1992;107(3):858-60. DOI:10.1111/j.1476-5381.1992.tb14537.x

29. Giaid A, Yanagisawa M, Langleben D, Michel R, Levy R, Shennib $\mathrm{H}$, et al. Expression of endothelin-1 in the lungs of patients with pulmonary hypertension. New England Journal of Medicine. 1993;328:1732-9.

30. Stewart DJ, Levy RD, Cernacek P, Langleben D. Increased Plasma Endothelin-1 in Pulmonary-Hypertension - Marker or Mediator of Disease. Annals of Internal Medicine. 1991;114(6):464-9. DOI:10.7326/0003-4819-114-6-464

31. Garg UC, Hassid A. Nitric oxide-generating vasodilators and 8-bromo-cyclic guanosine monophosphate inhibit mitogenesis and proliferation of cultured rat vascular smooth muscle cells. Journal of Clinical Investigation. 1989;83(5):1774-7. DOI:10.1172/JCI114081

32. Ignarro LJ, Buga GM, Wei LH, Bauer PM, Wu G, del Soldato P. Role of the arginine-nitric oxide pathway in the regulation of vascular smooth muscle cell proliferation. Proc Natl Acad Sci U S A. 2001;98(7):4202-8. DOI:10.1073/pnas.071054698

33. Tuder RM, Cool CD, Geraci MW, Wang J, Abman SH, Wright L, et al. Prostacyclin synthase expression is decreased in lungs from patients with severe pulmonary hypertension. American Journal of Respiratory Critical Care Medicine. 1999;159(6):1925-32.

34. Skalski J, Allison TG, Miller TD. The safety of cardiopulmonary exercise testing in a population with high-risk cardiovascular diseases. Circulation. 2012;126(21):2465-72. DOI:10.1161/CIRCULATIONAHA.112.110460

35. Linehan JH, Haworth ST, Nelin LD, Krenz GS, Dawson CA. A simple distensible vessel model for interpreting pulmonary vascular pressure-flow curves. Journal of applied physiology (Bethesda, Md 
: 1985). 1992;73(3):987-94.

36. Hillis LD, Firth BG, Winniford MD. Analysis of factors affecting the variability of fick versus indicator dilution measurements of cardiac output. The American Journal of Cardiology. 1985;56(12):7648. DOI:10.1016/0002-9149(85)91132-4

37. Hsu S, Brusca SB, Rhodes PS, Kolb TM, Mathai SC, Tedford RJ. Use of thermodilution cardiac output overestimates diagnoses of exercise-induced pulmonary hypertension. Pulmonary Circulation. 2017:690629-. DOI:10.1086/690629

38. Raeside Da, Smith a, Brown a, Patel KR, Madhok R, Cleland J, et al. Pulmonary artery pressure measurement during exercise testing in patients with suspected pulmonary hypertension. The European respiratory journal. 2000;16(2):282-7.

39. Sheriff DD, Augustyniak RA, O'Leary DS. Muscle chemoreflexinduced increases in right atrial pressure. American Journal of Physiology-Heart and Circulatory Physiology. 1998;275(3):H767H75. DOI:10.1152/ajpheart.1998.275.3.H767

40. Argiento $P$, Chesler N, Mulè M, D'Alto M, Bossone E, Unger P, et al. Exercise stress echocardiography for the study of the pulmonary circulation. European Respiratory Journal. 2010;35(6):1273-8. DOI:10.1183/09031936.00076009

41. Argiento $P$, Vanderpool RR, Mulè $M$, Russo MG, D'Alto $M$, Bossone $\mathrm{E}$, et al. Exercise stress echocardiography of the pulmonary circulation: Limits of normal and sex differences. Chest. 2012;142(5):1158-65. DOI:10.1378/chest.12-0071

42. McQuillan BM, Picard MH, Leavitt M, Weyman AE. Clinical correlates and reference intervals for pulmonary artery systolic pressure among echocardiographically normal subjects. Circulation. 2001;104(23):2797-802. DOI:10.1161/hc4801.100076

43. Bossone E, Naeije R. Exercise-Induced Pulmonary Hypertension. Heart Failure Clinics. 2012;8(3):485-95. DOI:10.1016/j.hfc.2012.04.007

44. Bossone E, Rubenfire M, Bach DS, Ricciardi M, Armstrong WF. Range of tricuspid regurgitation velocity at rest and during exercise in normal adult men: Implications for the diagnosis of pulmonary hypertension. Journal of the American College of Cardiology. 1999;33(6):1662-6. DOI:10.1016/S0735-1097(99)00055-8 45. Barst RJ, McGoon M, Torbicki A, Sitbon O, Krowka $\mathrm{MJ}$, Olschewski $\mathrm{H}$, et al. Diagnosis and differential assessment of pulmonary arterial hypertension. Journal of the American College of Cardiology. 2004;43(12 SUPPL.):40-8. DOI:10.1016/j.jacc.2004.02.032

46. Lewis GD, Shah RV, Pappagianopolas PP, Systrom DM, Semigran MJ. Determinants of ventilatory efficiency in heart failure: the role of right ventricular performance and pulmonary vascular tone. Circulation Heart failure. 2008;1(4):227-33. DOI:10.1161/CIRCHEARTFAILURE.108.785501

47. Kovacs G, Maier R, Aberer E, Brodmann M, Graninger W, Kqiku $X$, et al. Pulmonary arterial hypertension therapy may be safe and effective in patients with systemic sclerosis and borderline pulmonary artery pressure. Arthritis and rheumatism.
2012;64(4):1257-62. DOI:10.1002/art.33460

48. Saggar R, Khanna D, Furst DE, Shapiro S, Maranian P, Belperio $\mathrm{JA}$, et al. Exercise-induced pulmonary hypertension associated with systemic sclerosis: Four distinct entities. Arthritis and Rheumatism. 2010;62(12):3741-50. DOI:10.1002/art.27695

49. Schmeisser A, Schroetter H, Braun-Dulleaus RC. Management of pulmonary hypertension in left heart disease. Therapeutic advances in cardiovascular disease. 2013;7(3):131-51. DOI:10.1177/1753944713477518

50. Ho JE, Zern EK, Lau ES, Wooster L, Bailey CS, Cunningham $\mathrm{T}$, et al. Exercise Pulmonary Hypertension Predicts Clinical Outcomes in Patients With Dyspnea on Effort. Journal of the American College of Cardiology. 2020;75(1):17-26. DOI:10.1016/j.jacc.2019.10.048

51. Parasuraman S, Walker S, Loudon BL, Gollop ND, Wilson AM, Lowery $\mathrm{C}$, et al. Assessment of pulmonary artery pressure by echocardiography-A comprehensive review. IJC Heart Vasculature. 2016;12:45-51. DOI:10.1016/j.ijcha.2016.05.011 
1. $\mathrm{PAH}$

1.1 Idiopathic PAH

1.2. Heritable PAH

1.3. Drug- and toxin-induced $\mathrm{PAH}$

1.4. $\mathrm{PAH}$ associated with:

1.4.1. Connective tissue disease

1.4.2. HIV infection

1.4.3. Portal hypertension

1.4.4. Congenital heart disease

1.4.5. Schistosomiasis

1.5. PAH long-term responders to calcium channel blockers

1.6. $\mathrm{PAH}$ with overt features of venous/capillaries (PVOD/PCH) involvement

1.7. Persistent $\mathrm{PH}$ of the newborn syndrome

2. $\mathrm{PH}$ due to left heart disease

2.1. $\mathrm{PH}$ due to heart failure with preserved LVEF

2.2. $\mathrm{PH}$ due to heart failure with reduced LVEF

2.3. Valvular heart disease

2.4. Congenital/acquired cardiovascular conditions leading to post-capillary $\mathrm{PH}$

3. $\mathrm{PH}$ due to lung diseases and/or hypoxia

3.1. Obstructive lung disease

3.2. Restrictive lung disease

3.3. Other lung disease with mixed restrictive/obstructive pattern

3.4. Hypoxia without lung disease

3.5. Developmental lung disorders

4. $\mathrm{PH}$ due to pulmonary artery obstructions

4.1. Chronic thromboembolic $\mathrm{PH}$

4.2. Other pulmonary artery obstructions

5. $\mathrm{PH}$ with unclear and/or multifactorial mechanisms

5.1. Hematological disorders

5.2. Systemic and metabolic disorders

5.3. Others

5.4. Complex congenital heart disease

LVEF, left ventricular ejection fraction; $\mathrm{PAH}$, pulmonary arterial hypertension; $\mathrm{PCH}$, pulmonary capillary hemangiomatosis; $\mathrm{PH}$, pulmonary hypertension; PVOD, pulmonary veno-occlusive disease. Reproduced from Simonneau et al. under Creative Commons Attribution-NonCommercial 4.0 International (CC BY-NC 4.0). (9)

TABLE 1 Updated clinical classification of pulmonary hypertension 


\begin{tabular}{l|l} 
Definition & Characteristics \\
\hline Pulmonary hypertension & Resting mPAP $20 \mathrm{mmHg}$ \\
\hline Exercise Induced pulmonary hypertension & Resting PVR 3 Wood Units \\
& $\mathrm{mPAP} / \mathrm{CO}$ slope $>3 \mathrm{mmHg} / \mathrm{L} / \mathrm{min}$ during exercise \\
& mPAP $>30 \mathrm{mmHg}$ with a PVR $>3$ Wood Units during exercise
\end{tabular}

TAB LE 2 Proposed hemodynamic definitions of pulmonary hypertension and exercise induced pulmonary hypertension 